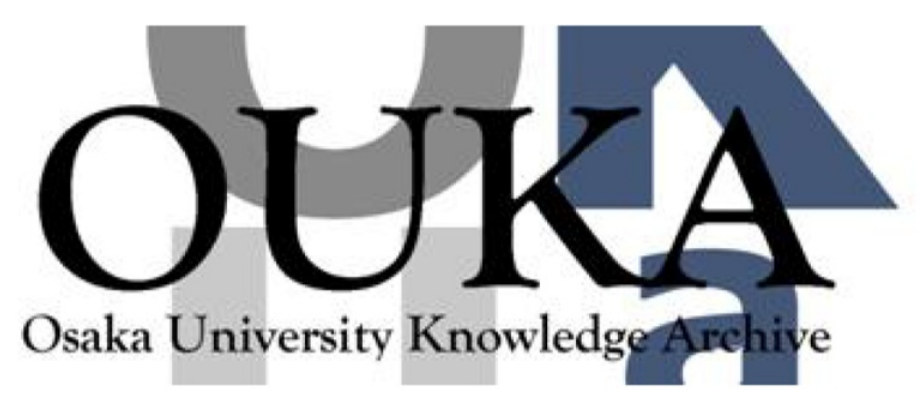

\begin{tabular}{|c|c|}
\hline Title & $\begin{array}{l}\text { Rigid cyclic polymer in solution: Cycloamylose } \\
\text { tris(phenylcarbamate) in 1,4-dioxane and 2- } \\
\text { ethoxyethanol }\end{array}$ \\
\hline Author (s) & $\begin{array}{l}\text { Terao, Ken; Asano, Natsuki; Kitamura, Shinichi } \\
\text { et al. }\end{array}$ \\
\hline Citation & ACS Macro Letters. 1(11) p. 1291-p. 1294 \\
\hline Issue Date & $2012-10-18$ \\
\hline oaire:version & AM \\
\hline URL & https://hdl. handle. net/11094/81804 \\
\hline rights & $\begin{array}{l}\text { This document is the Accepted Manuscript } \\
\text { version of a Published Work that appeared in } \\
\text { final form in ACS Macro Letters, } \odot \text { American } \\
\text { Chemical Society after peer review and } \\
\text { technical editing by the publisher. To access } \\
\text { the final edited and published work see } \\
\text { https://doi.org } / 10.1021 / \mathrm{mz} 3004506 \text {. }\end{array}$ \\
\hline Note & \\
\hline
\end{tabular}

Osaka University Knowledge Archive : OUKA

https://ir. Library. osaka-u. ac. jp/

Osaka University 


\title{
Rigid Cyclic Polymer in Solution: Cycloamylose tris(phenylcarbamate) in 1,4-Dioxane and 2-Ethoxyethanol
}

\author{
Ken Terao, ${ }^{*, \dagger}$ Natsuki Asano, ${ }^{\dagger}$ Shinichi Kitamura, ${ }^{\ddagger}$ and Takahiro Sato ${ }^{\dagger}$ \\ tDepartment of Macromolecular Science, Graduate School of Science, Osaka University, 1-1. Machikaneyama-cho, \\ Toyonaka, Osaka 560-0043, Japan \\ ${ }^{*}$ Graduate School of Life and Environmental Sciences, Osaka Prefecture University, Gakuen-cho, Nakaku, Sakai, \\ Osaka 599-8531, Japan \\ RECEIVED DATE (automatically inserted by publisher); kterao@chem.sci.osaka-u.ac.jp
}

\begin{abstract}
Six cyclic amylose tris(phenylcarbamate) (ATPC) samples have been prepared from enzymatically synthesized cyclic amylose ranging in the number of repeat units $N$ from 24 to 290. Synchrotron-radiation small-angle X-ray scattering measurements were made for the samples in 1,4-dioxane (DIOX) and 2-ethoxyethanol (2EE) to determine the $z$-average radius of gyration $\left\langle S^{2}\right\rangle_{\mathrm{z}}$ and the particle scattering function $P(q)$. Molar mass dependencies of $\left\langle S^{2}\right\rangle_{z}$ in the two solvents were successfully explained by the current theories for the wormlike ring with the same parameters for linear ATPC in the corresponding solvent, that is, the helix pitch $h$ (or contour length) per residue and the Kuhn segment length $\lambda^{-1}$ (stiffness parameter, twice of the persistence length). The latter parameter $\lambda^{-1}$ are 22 and $16 \mathrm{~nm}$ in DIOX and 2EE, respectively. Except for the low- $q$ region, $P(q)$ was also explained by the rigid ring having the same contour length $\mathrm{Nh}$ as that for linear ATPC. Further, their local conformation estimated from circular dichroism spectra is essentially unaltered from that for linear ATPC at least when $N \geq 24$.
\end{abstract}
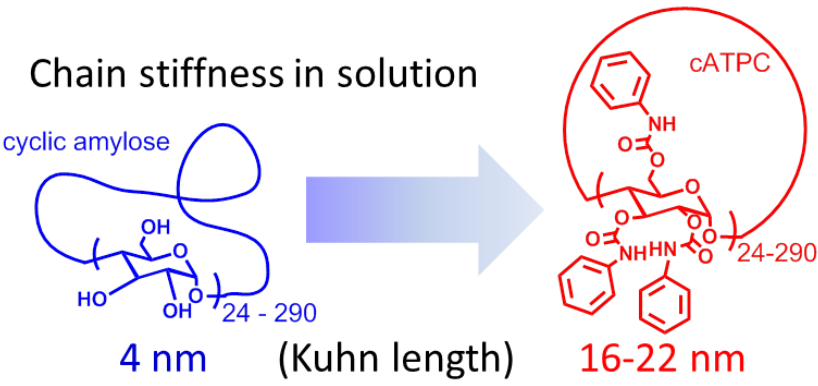

Cyclic or ring polymers have a topologically interesting structure with no chain ends, and their dimensional and also various physical properties have attracted much attention from both theoretical and experimental points of view. After pioneering works for the synthetic ring polymers, ${ }^{1}$ dimensional and hydrodynamic properties of ring polystyrene, ${ }^{2}$ polydimethylsiloxane, ${ }^{3}$ amylose, ${ }^{4}$ and DNA $^{5}$ have been extensively studied in the past few decades.

It is well known that the chain stiffness is a decisively important factor affecting dimensional and physical properties of linear-chain polymers. ${ }^{6}$ Therefore, the chain stiffness may play an important role also in the properties of cyclic polymers. However, experimental studies of rigid ring polymers were rare due to difficulty in their synthesis even though various ring polymers were recently synthesized. ${ }^{7}$ Because of low ring closure probability, it is difficult to transform stiff linear polymers into cyclic ones. While double helical cyclic DNA has a stiff main chain, it may not be a very good example because of its superhelical conformation in solution. ${ }^{5}$

Another strategy to obtain rigid ring is thus to stiffen flexible ring by using some appropriate chemical modification. In actuality, some macrocyclic brush polymers are reported very recently, ${ }^{8}$ and they may have rather stiff main chain due to the repulsion force between side chains since it is well-known that the main chain of comb polymers having high graft density has stiffer main chain than that for the corresponding linear chain. However, the stiffening efficiency is not very high even though the high grafting density. ${ }^{9}$

Amylose tris(phenylcarbamate) (ATPC) has much stiffer main chain $^{10}$ in several organic solvents than amylose in dimethyl sulfoxide. ${ }^{11}$ Fortunately, this polymer can be synthesized quantitatively without main chain scission when the molar mass of amylose is below $10^{6} .{ }^{12}$ Furthermore, cyclic amylose having more than 100 pyranose can be synthesized enzymatically. ${ }^{4 c}$ The sample has high purity and it behaves as rather flexible polymer as is the case with linear amylose. ${ }^{4}$

We thus synthesized cyclic amylose tris(phenylcarbamate) (cATPC) from four cyclic amylose samples of which weightaverage molar mass $M_{\mathrm{w}}$ ranges from $3.1 \times 10^{3}$ to $2.8 \times 10^{4}$, in the manner for linear ATPC samples. ${ }^{12}$ The resultant samples were purified and extensively fractionated by precipitation method to obtain six cATPC samples. Both their solution NMR and solid state IR spectra were essentially the same as those for our ATPC samples, and furthermore the degree of substitution was estimated to be $3.0 \pm 0.2$ from the ratio of carbon to nitrogen estimated from elemental analysis, indicating full substitution of amylose. This is consistent with our recent work for linear ATPC, but therefore 
mass spectroscopy methods cannot be applicable to determine the linkages unlike other synthetic ring polymers. The dispersity index (ratio of $M_{\mathrm{w}}$ to the number average molar mass $M_{\mathrm{n}}$ ) was determined to be between 1.05 and 1.2 from size exclusion chromatography equipped with two-angle $\left(7^{\circ}\right.$ and $\left.90^{\circ}\right)$ light scattering photometer. Their circular dichroism spectra in 1,4dioxane (DIOX) are presented in Figure 1. See ref. 12 for experimental details. Both the molar elipticity $\Delta \varepsilon$ and the molar absorption coefficient $\varepsilon$ for cATPC20K (and for cATPC13K, not shown here) are essentially the same as those for linear polymer, ATPC $200 \mathrm{~K}$, indicating that local conformation of cATPC is essentially the same as those for linear polymer.

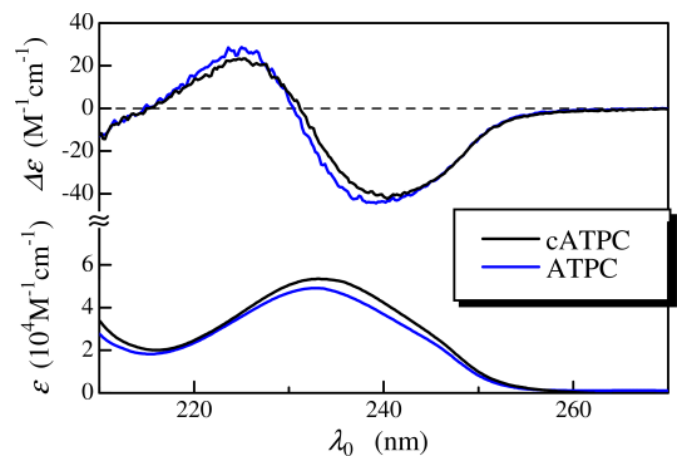

Figure 1. Comparison between circular dichroism spectra for cATPC20K and ATPC200K samples in DIOX both at $25^{\circ} \mathrm{C}$.

Synchrotron-radiation small-angle X-ray scattering (SAXS) measurements were made on the cATPC samples in DIOX and 2ethoxyethanol (2EE) at $25^{\circ} \mathrm{C}$. The experiments were carried out at the BL40B2 beamline in SPring-8 and the BL-10C beamline in KEK-PF. The wavelength, camera length, and accumulation time were chosen to be $0.10-0.15 \mathrm{~nm}, 1500-4000 \mathrm{~mm}$, and 300 sec, respectively. See ref. 12 for experimental details. The Guinier plot was utilized to determine $M_{\mathrm{w}}$, the particle scattering function $P(q)$, and $z$-average radius of gyration $\left\langle S^{2}\right\rangle_{z}$. It should be noted that a previously investigated ATPC sample (ATPC20K) $)^{12}$ was used as the standard of the molar mass. The resultant $M_{\mathrm{w}}$ and $\left\langle S^{2}>_{\mathrm{z}}{ }^{1 / 2}\right.$ are summarized in Table 1. It should be noted that we chose these two solvents, DIOX and 2EE, because of the larger number of intramolecular hydrogen bonds for ATPC in DIOX compared with that in 2EE. ${ }^{12}$

Table 1. Weight-average molar mass $M_{\mathrm{w}}$ and $z$-average radius of gyration $\left\langle S^{2}\right\rangle_{z}{ }^{1 / 2}$ for CATPC in DIOX and 2EE at $25^{\circ} \mathrm{C}$.

\begin{tabular}{cccc}
\hline \multirow{2}{*}{ Sample } & $M_{\mathrm{w}} / 10^{4}$ & \multicolumn{2}{c}{$<S^{2}>_{\mathrm{z}}^{1 / 2}(\mathrm{~nm})$} \\
\cline { 3 - 4 }$\left(\mathrm{g} \mathrm{mol}^{-1}\right)$ & in DIOX & in 2EE \\
\hline cATPC13K & 1.25 & 1.65 & 1.55 \\
сATPC20K & 1.83 & 2.2 & 2.0 \\
сAТРC40K & 4.46 & 4.6 & 3.8 \\
сATPC50K & 4.73 & 5.1 & 4.3 \\
сATPC80K & 8.19 & 7.4 & 5.9 \\
cАТPC150K & 14.9 & 11.0 & 9.8 \\
\hline
\end{tabular}

Figure 2 shows the molar mass dependence of $\left\langle S^{2}\right\rangle_{\mathrm{z}}^{1 / 2}$ for cATPC and ATPC in the two solvents. The obtained $<S^{2}>_{\mathrm{z}}^{1 / 2}$ for the cyclic polymer in each solvent is $60-65 \%$ as small as that for the linear ATPC and the slope is almost the same as that for linear one, indicating that $\mathrm{ATPC}$ behaves as rather rigid ring in solution. Since dimensional properties for ATPC, ${ }^{10,12-13}$ amylose tris(3,5dimethylpheylcarbamate), ${ }^{14}$ amylose tris $(n$-butylcarbamate $),{ }^{15}$ and amylose-2-acetyl-3,6-bis(phenylcarbamate) ${ }^{16}$ are well explained by the current theories for the Kratky-Porod wormlike chain, ${ }^{17}$ cyclic wormlike chain may be a good model for cATPC.

According to Shimada et al., theoretical radius of gyration $\left\langle S^{2}>_{0}\right.$ for cyclic wormlike chain is calculated to be ${ }^{18}$

$$
\begin{aligned}
& \left\langle S^{2}\right\rangle_{0}=\frac{L^{2}}{4 \pi^{2}}\left[\begin{array}{l}
1-0.1140 \lambda L-0.0055258(\lambda L)^{2} \\
+0.0022471(\lambda L)^{3}-0.00013155(\lambda L)^{4}
\end{array}\right] \text { for } \lambda L \leq 6 \\
& =\frac{L}{12 \lambda}\left\{1-\frac{7}{6 \lambda L}-0.025 \exp \left[-0.01(\lambda L)^{2}\right]\right\} \quad \text { for } \quad \lambda L \geq 6
\end{aligned}
$$

where $\lambda^{-1}$ is the Kuhn segment length and $L$ is the contour length which is related with molar mass $M$ as

$$
L=M / M_{\mathrm{L}}
$$

where $M_{\mathrm{L}}$ is the molar mass per unit contour length. In this case, chain thickness may not be negligible. Since gyration radius for cylindrical rigid ring is calculated to be

$$
\left\langle S^{2}\right\rangle=\frac{L^{2}}{4 \pi^{2}}+\frac{d^{2}}{4}
$$

where $d$ denotes the chain diameter. The $<S^{2}>$ for cylindrical wormlike ring was calculated by the sum of $\left\langle S^{2}>_{0}\right.$ and $d^{2} / 4$. The three parameters were determined from the curve-fitting procedure to be $M_{\mathrm{L}}=1450 \pm 100 \mathrm{~nm}^{-1}, \lambda^{-1}=22 \pm 3 \mathrm{~nm}$, and $d=$ $1.7 \pm 0.2 \mathrm{~nm}$ in DIOX and $M_{\mathrm{L}}=1550 \pm 100 \mathrm{~nm}^{-1}, \lambda^{-1}=16 \pm 3 \mathrm{~nm}$, and $d=1.6 \pm 0.2 \mathrm{~nm}$ in $2 \mathrm{EE}$; they are comparable with those for linear ATPC. ${ }^{12}$ The calculated theoretical solid lines reproduce well the experimental data and furthermore the theoretical values for rather flexible ring of which chain stiffness is the same as cyclic amylose $\left(\lambda^{-1}=4 \mathrm{~nm}\right)^{4 \mathrm{c}}$ are substantially smaller than the experimental data at higher $M$ range, confirming much higher rigidity of cATPC compared with cyclic amylose in solution. It should be noted that intramolecular excluded volume effects may be negligibly small since it is not significant for linear ATPC even for much higher $M_{\mathrm{w}}$ samples $\left(<3 \times 10^{6}\right) .^{12}$ 


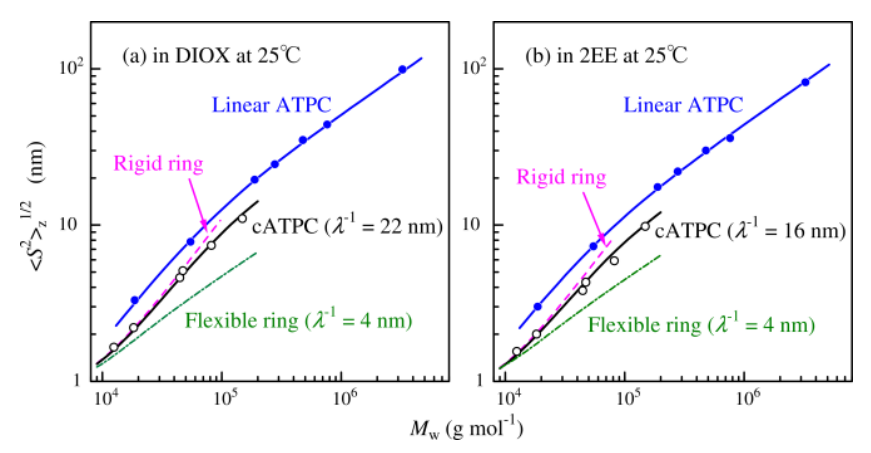

Figure 2. Molar mass dependence of $z$-average radius of gyration $\left\langle S^{2}\right\rangle_{\mathrm{z}}^{1 / 2}$ for cATPC (open circles) and linear ATPC ${ }^{12}$ (filled cirlcles) in 1,4dioxane (DIOX, a) and 2-ethoxyethanol (2EE, b) both at $25{ }^{\circ} \mathrm{C}$. Solid blue lines, theoretical values for cyclic wormlike cylinder with the parameters in the text. Dashed line (magenta), $\left\langle S^{2}>^{1 / 2}\right.$ for rigid ring. Dotdashed line (green), $<S^{2}>^{1 / 2}$ for wormlike cyclic cylinder with $\lambda^{-1}=4 \mathrm{~nm}$.

The particle scattering function $P(q)$ for cATPC is displayed in Figure 3 in the form of the Holtzer plot, where $q$ is the magnitude of the scattering vector. In panels (a) and (b), $M_{\mathrm{w}} q P(q)$ for cATPC $50 \mathrm{~K}$ and $\mathrm{cATPC} 20 \mathrm{~K}$ is compared with that for linear ATPC samples having close $M_{\mathrm{w}}$, that is, ATPC50K $\left(M_{\mathrm{w}}=5.48 \times\right.$ $\left.10^{4}\right)$ and ATPC20K $\left(M_{\mathrm{W}}=1.87 \times 10^{4}\right){ }^{12}$ While so-called Holtzer plateau region is seen for linear ATPC $\left(0.3 \mathrm{~nm}^{-1}<q<1 \mathrm{~nm}^{-1}\right)$, an appreciable clear peak is found for each cATPC sample, and $M_{\mathrm{w}} q P(q)$ at higher $q$ region are the same as each other. This is likely because cATPC has smaller $\left\langle S^{2}\right\rangle_{\mathrm{z}}$ than that for the linear ATPC having the same molecular weight and the local conformation of ring and linear polymers is comparable to each other.

Although the theoretical calculations of $P(q)$ for wormlike ring have not been published, the experimental data for lower $M_{\mathrm{w}}$ samples may be compared with the theoretical value for rigid cylindrical rings

$P(q)=\int_{0}^{\pi / 2}\left[4 J_{0}\left(\frac{L q \sin \xi}{2 \pi}\right) J_{1}\left(\frac{d q}{2}\right) / d q\right]^{2} \sin \xi d \xi$

since their experimentally obtained $\left\langle S^{2}\right\rangle_{\mathrm{z}}$ are almost equivalent to the rigid ring, shown as dashed lines in Figure 2. Here, $J_{\mathrm{n}}(x)$ is the Bessel function of the $n$-th order. Theoretical green dotdashed curves in Figure 3 calculated for rigid rings having $M_{\mathrm{L}}=$ $1600 \pm 50 \mathrm{~nm}^{-1}$ and $d=1.6 \pm 0.1 \mathrm{~nm}$ in DIOX, and $M_{\mathrm{L}}=1640 \pm$ $60 \mathrm{~nm}^{-1}$ and $d=1.5 \pm 0.1 \mathrm{~nm}$ in 2EE fairly fit the experimental data for lower two $M_{\mathrm{w}}$ samples and the higher $q$ range for the other four samples, but the theoretically obtained fluctuation cannot be seen in the experimental data. This is likely due to polydispersity of the CATPC samples as well as the finite rigidity of the real cyclic chains. In fact, the solid red and dashed blue curves calculated for rigid rings with $M_{\mathrm{w}} / M_{\mathrm{n}}=1.05$ and 1.2 , respectively, for which the polydispersity was considered by using the log normal distribution, mostly reproduce the experimental data except for the lower $q$ range for four higher $M_{\mathrm{w}}$ samples. This underestimated theoretical $P(q)$ should be due to the finite chain stiffness of cATPC. Indeed, the discrepancy becomes more significant for 2EE solution than that in DIOX, and furthermore it increases with increasing $M_{\mathrm{w}}$. Considering the obtained $M_{\mathrm{L}}$ and $d$ are comparable to those for $\left\langle S^{2}\right\rangle_{\mathrm{z}}$, we may conclude that both the $P(q)$ and $\left\langle S^{2}\right\rangle_{\mathrm{z}}$ data are consistently explained by the current theories.

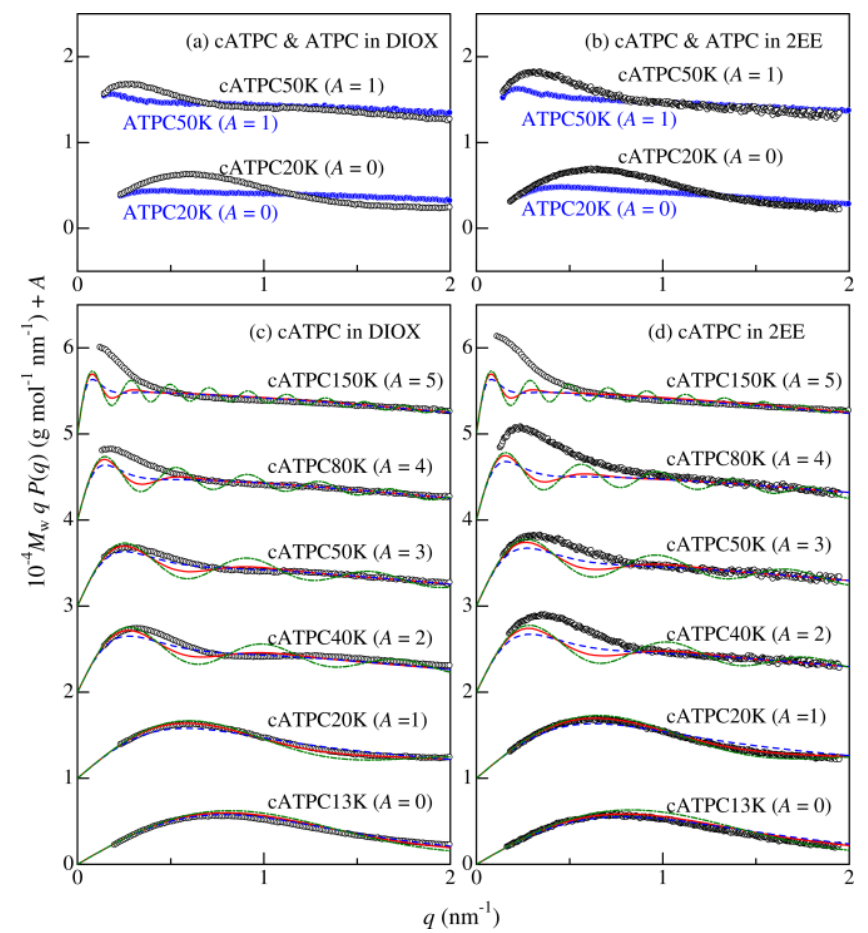

Figure 3. Reduced Holtzer plots for indicated cATPC samples in DIOX (a) and 2EE (b) all at $25^{\circ} \mathrm{C}$. Green dot-dashed, red solid, and blue dashed curves, theoretical values for polydisperse rigid rings with $M_{\mathrm{w}} / M_{\mathrm{n}}$ of 1 , 1.05 , and 1.2 , respectively. The ordinate values are shifted by $A$ for clarity.

The obtained wormlike chain parameters for cATPC are summarized along with those for ATPC ${ }^{12}$ in Table 2, in which the helix pitch per residue $h$ was calculated by $h=M_{0} / M_{\mathrm{L}}$, where $M_{0}$ is the molar mass of the repeat unit. While the chain stiffness in DIOX is appreciably higher than that in 2EE, both parameters for cATPC are mostly equivalent to those for ATPC. Considering also essentially the same circular dichroism spectra for cATPC and linear ATPC (Table 1), both the local conformation and the chain stiffness of cATPC in the two solvents are essentially the same as those for the linear ATPC in the same solvent. In other words, we successfully obtained quite stiff cyclic polymer consisting of $24-290$ repeat units, corresponding to $8-100 \mathrm{~nm}$ in the contour length. Thus, we may conclude that cATPC is a good model compound as rigid ring polymer. Very recently, we reported much stiffer amylose derivatives, that is, amylose tris $(n$ butylcarbamate) $\left(\lambda^{-1}=75 \mathrm{~nm} \text { in tetrahydrofuran }\right)^{15 \mathrm{a}}$ and amylose tris (3,5-dimethylphenylcarbamate) $\left(\lambda^{-1}=73 \mathrm{~nm}\right.$ in 4-methyl-2pentanone), ${ }^{14}$ suggesting more rigid ring polymers might be obtained from the similar procedure. 
Table 2. Values of the Helix Pitch per Residue $h$, the Kuhn Segment Length $\lambda^{-1}$, and the Chain Diameter $d$ for CATPC and ATPC in DIOX and 2EE at $25^{\circ} \mathrm{C}$.

\begin{tabular}{ccccc}
\hline Polymer & Solvent & $h(\mathrm{~nm})$ & $\lambda^{-1}(\mathrm{~nm})$ & $d(\mathrm{~nm})$ \\
\hline cATPC & DIOX & $0.34 \pm 0.02$ & $22 \pm 3$ & $1.6 \pm 0.1$ \\
ATPC $^{\text {a }}$ & DIOX & $0.34 \pm 0.01$ & $22 \pm 2$ & 1.5 \\
cATPC $^{2}$ & $2 \mathrm{EE}$ & $0.33 \pm 0.02$ & $16 \pm 3$ & $1.5 \pm 0.2$ \\
ATPC $^{\text {a }}$ & $2 \mathrm{EE}$ & $0.32 \pm 0.01$ & $16 \pm 2$ & 1.3 \\
$\operatorname{Ref}^{12}$ & & & &
\end{tabular}

\section{ASSOCIATED CONTENT Supporting Information}

Detailed explanation of experimental procedures including synthesis, fractional precipitation, and size exclusion chromatography. Guinier plots. This material is available free of charge via the Internet at http://pubs.acs.org

\section{AUTHOR INFORMATION \\ Corresponding Author \\ *E-mail: kterao@chem.sci.osaka-u.ac.jp Notes \\ The authors declare no competing financial interest.}

\section{ACKNOWLEDGMENTS}

The synchrotron radiation experiments were performed at the BL40B2 in SPring-8 with the approval of the Japan Synchrotron Radiation Research Institute (JASRI) (Proposal Nos. 2010B1126 and 2011A1049) and at the BL-10C in KEK-PF under the approval of the Photon Factory Program Advisory Committee (No. 2010G080). The authors thank Prof. Yoji Inoko (Osaka Univ.) for SAXS measurements in KEK-PF. This work was partially supported by Grant-in-Aid for Young Scientists (\#23750128) from Japan Society for the Promotion of Science (JSPS).

\section{References}

(1)(a)Dodgson, K.; Sympson, D.; Semlyen, J. A. Polymer 1978, 19, 12851289; (b)Roovers, J.; Toporowski, P. M. Macromolecules 1983, 16, 843849; (c)McKenna, G. B.; Hostetter, B. J.; Hadjichristidis, N.; Fetters, L. J.; Plazek, D. J. Macromolecules 1989, 22, 1834-1852; (d)Takaha, T.; Yanase, M.; Takata, H.; Okada, S.; Smith, S. M. J. Biol. Chem. 1996, 271, 2902-2908.

(2)(a)Ragnetti, M.; Geiser, D.; Hocker, H.; Oberthur, R. C. Makromol. Chem. Macromol. Chem. Phys. 1985, 186, 1701-1709; (b)Lutz, P.; McKenna, G. B.; Rempp, P.; Strazielle, C. Makromolekulare ChemieRapid Communications 1986, 7, 599-605; (c)Hadziioannou, G.; Cotts, P. M.; Tenbrinke, G.; Han, C. C.; Lutz, P.; Strazielle, C.; Rempp, P.; Kovacs, A. J. Macromolecules 1987, 20, 493-497; (d)Takano, A.; Ohta, Y.; Masuoka, K.; Matsubara, K.; Nakano, T.; Hieno, A.; Itakura, M.; Takahashi, K.; Kinugasa, S.; Kawaguchi, D.; Takahashi, Y.; Matsushita, Y. Macromolecules 2012, 45, 369-373.

(3) Higgins, J. S.; Dodgson, K.; Semlyen, J. A. Polymer 1979, 20, 553558.

(4)(a)Kitamura, S.; Isuda, H.; Shimada, J.; Takada, T.; Takaha, T.; Okada, S.; Mimura, M.; Kajiwara, K. Carbohydrate Research 1997, 304, 303314; (b)Shimada, J.; Kaneko, H.; Takada, T.; Kitamura, S.; Kajiwara, K. Journal of Physical Chemistry B 2000, 104, 2136-2147; (c)Nakata, Y.; Amitani, K.; Norisuye, T.; Kitamura, S. Biopolymers 2003, 69, 508-516. (5)(a)Brady, G. W.; Fein, D. B.; Lambertson, H.; Grassian, V.; Foos, D.; Benham, C. J. Proc. Natl. Acad. Sci. U. S. A. 1983, 80, 741-744; (b)Langowski, J.; Giesen, U. Biophysical Chemistry 1989, 34, 9-18; (c)Seils, J. C.; Pecora, R. Macromolecules 1992, 25, 354-362; (d)Fishman, D. M.; Patterson, G. D. Biopolymers 1996, 38, 535-552.
(6) Norisuye, T. Prog. Polym. Sci. 1993, 18, 543-584.

(7)(a)Endo, K. In New Frontiers in Polymer Synthesis; Kobayashi, S., Ed.; Springer-Verlag Berlin: Berlin, 2008; Vol. 217, p 121-183; (b)Laurent, B. A.; Grayson, S. M. Chem. Soc. Rev. 2009, 38, 2202-2213; (c)Kricheldorf, H. R. J. Polym. Sci., Part A: Polym. Chem. 2010, 48, 251-284; (d) Yamamoto, T.; Tezuka, Y. Polym. Chem. 2011, 2, 1930-1941.

(8)(a)Schappacher, M.; Deffieux, A. Science 2008, 319, 1512-1515; (b)Lahasky, S. H.; Serem, W. K.; Guo, L.; Garno, J. C.; Zhang, D. H. Macromolecules 2011, 44, 9063-9074; (c)Zhang, K.; Tew, G. N. ACS Macro Lett. 2012, 1, 574-579.

(9)(a)Zhang, B.; Groehn, F.; Pedersen, J. S.; Fischer, K.; Schmidt, M. Macromolecules 2006, 39, 8440-8450; (b)Nakamura, Y.; Norisuye, T. In Soft-Matter Characterization; Borsali, R., Pecora, R., Eds.; Springer: Berlin, 2008; Vol. 1, p 236-286.

(10) Burchard, W. In Soft-Matter Characterization; Borsali, R.

Pecora, R. ed.; Springer: Berlin, 2008; Vol. 1, p 465-603.

(11) Nakanishi, Y.; Norisuye, T.; Teramoto, A.; Kitamura, S Macromolecules 1993, 26, 4220-4225.

(12) Terao, K.; Fujii, T.; Tsuda, M.; Kitamura, S.; Norisuye, T. Polym. J. 2009, 41, 201-207.

(13) Fujii, T.; Terao, K.; Tsuda, M.; Kitamura, S.; Norisuye, T. Biopolymers 2009, 91, 729-736.

(14) Tsuda, M.; Terao, K.; Nakamura, Y.; Kita, Y.; Kitamura, S.; Sato, T. Macromolecules 2010, 43, 5779-5784.

(15)(a)Terao, K.; Murashima, M.; Sano, Y.; Arakawa, S.; Kitamura, S.; Norisuye, T. Macromolecules 2010, 43, 1061-1068; (b)Sano, Y.; Terao, K.; Arakawa, S.; Ohtoh, M.; Kitamura, S.; Norisuye, T. Polymer 2010, 51, 4243-4248; (c)Arakawa, S.; Terao, K.; Kitamura, S.; Sato, T. Polym. Chem. 2012, 3, 472-478.

(16) Tsuda, M.; Terao, K.; Kitamura, S.; Sato, T. Biopolymers 2012, 97, 1010-1017.

(17) Kratky, O.; Porod, G. Recl. Trav. Chim. Pays-Bas 1949, 68, 11061122 .

(18) Shimada, J.; Yamakawa, H. Biopolymers 1988, 27, 657-673. 
For Table of Contents Use Only

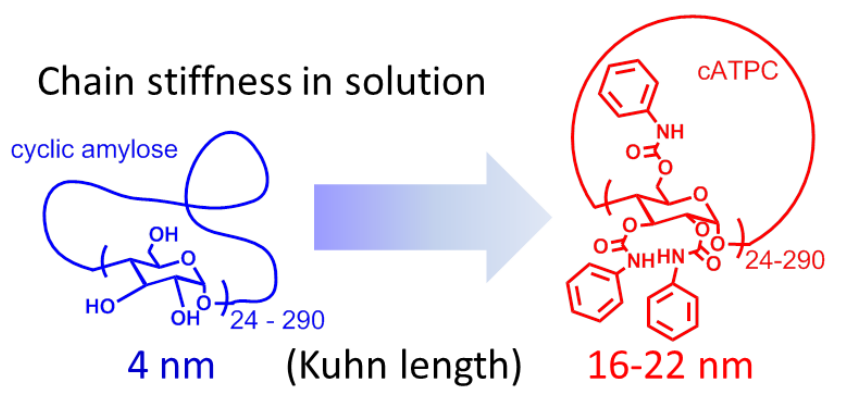

Rigid Cyclic Polymer in Solution: Cycloamylose tris(phenylcarbamate) in 1,4-Dioxane and 2-Ethoxyethanol Ken Terao,* Natsuki Asano, Shinichi Kitamura, Takahiro Sato 